\title{
On Almost WJCP-Injective Rings
}

\author{
R.D. Mahmood \\ Sh.H. Ahmad \\ Mathematics Department, College of Computers Sciences and \\ Mathematics, University of Mosul, Mosul, IRAQ
}

\author{
حول الحلقات الغامرة من النمط - WJCP تقريباً \\ م مشيماء حاتم أحمد \\ أ. أد. رائدة داؤد محمود \\ قسم الرياضيات \\ كلية علوم الحاسوب والرياضيات \\ جامعة الموصل
}

Received

$10 / 06 / 2018$
Accepted

07 / 11 / 2018

\section{الخلاصة}

يقال للحلقة R بأنها غامرة من النمط - WJCP تقريباً إذا كان لكل (مثالي ايمن غير منفرد)

bÆY(R)

مميزات وخواص الحلقات الغامرة من النمط - WJCP تقريباً والذي هو تعميم للحلقات الغامرة من

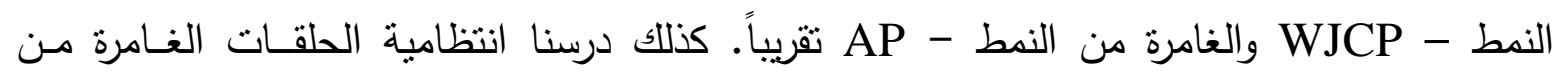

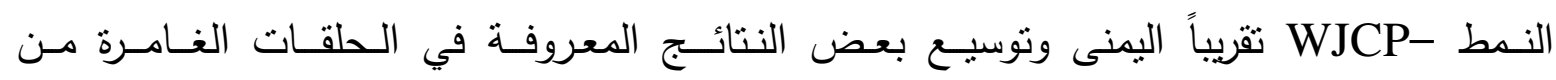

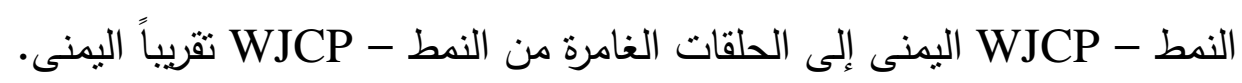

\section{ABSTRACT}

Let $\mathrm{R}$ be a ring. The ring $R$ is called right almost WJCP-injective. If for any $b \notin Y(R)$, (right non singular) there exists a left ideal $X_{b}$ of $R$ such that $l_{R} r_{R}(b)=R b \oplus X_{b}$. In this paper, we give some characterization and properties of almost WJCP-injective rings, which are proper generalization of JCP-injective ring and almost AP-injective ring. Then, we study the regularity of the right almost WJCP-injective ring and some important results which are known for the right JCP-injective rings to be hold for the right almost WJCPinjective rings. 
Keywords: JCP-injective rings, almost nil-injective rings, quasi regular rings and reduced rings.

\section{1- Introduction}

In this paper, $R$ will be an associative ring with identity and all modules are unitary right $R$-modules. For subset $X$ of $R$, the right (left) annihilator of $X$ in $R$ is denoted by $r(X)(l(X))$. If $X=\{a\}$, we usually abbreviate $r(a)$ and $l(a)$ for any a $\in \mathrm{R}$. We write $J(R), N(R), Y(R),(Z(R))$ for the Jacobson radical, the set of nilpotent elements, and right (left) singular ideal of $R$, respectively.

At first, we recall that a ring $R$ is called right principally injective [4] (or P-injective for short), if every homomorphism from a principally right ideal of $R$ to $R$ can be extended to an endomorphism of $R$, or equivalently $l(r(a))=R a$ for all $a \in R$. The notion of the right P-injective rings has been generalized by many authors see ([2], [5]). In ([12], [14]) the right P-injective rings are almost principally injective rings, a ring $R$ is said to be almost principally injective (or AP-injective for short), if for any $a \in R$, there exists a left ideal $X_{a}$ such that $l(r(a))=R a \oplus X_{a}$.

Von Neumann regular rings have been studied extensively by many authors (for example [3]). It is well known that a ring $R$ is regular if for any $a \in R$, there exists $b \in R$ such that $a=a b a$.

In [7] JCP-injective rings are studied. A ring $R$ is called a right JCPinjective, if for any right nonsingular element $c$ of $R$ and any $R$-homomorphism $g: c R \rightarrow R$, there exists $m \in R$ such that $g(c a)=m c a$ for all $a \in R$. Cleary, the right $\mathrm{P}$-injective rings are right JCP-injective rings. The nice structure of JCP-injective rings draws our attention to define almost WJCPinjective rings (or the right AWJCP-injective rings), and to investigate their characterizations and properties.

A ring $R$ is called reduced, if $N(R)=0$. A ring $R$ is said to be a biregular ring if for any $a \in R, R a R$ is generated by central idempotent [11]. In [8] the module $M$ is called almost principally a small injective (or APSinjective for short) if for any $a \epsilon J(R)$, there exists an S-submodule $X_{a}$ of $M$ such that $l_{M}(r(a))=M a \oplus X_{a}$ as left S-module. If $R_{R}$ is an APS-injective module, then we call $R$ a right APS-injective.

\section{2- Characterizations of Right AWJCP-Injective Rings}

In this section, we shall study characterizations of right AWJCPinjective rings.

\section{Definition 2.1}

Let $M_{R}$ be a module with $S=\operatorname{End}\left(M_{R}\right)$. The module $M$ is called right almost WJCP-injective (AWJCP-injective), if for any $a \in R \backslash Y(R)$, there exists an S-submodule $X_{a}$ of $M$ such that $l_{M} r_{R}(a)=M a \oplus X_{a}$ as left S-module. If 
$R_{R}$ is almost WJCP-injective, then we call $R_{R}$ a right almost WJCP-injective ring.

Every AP-injective ring is AWJCP-injective but the converse is not true. [Example 2.4, 7]

\section{Theorem 2.2}

Let $\left\{X_{a}: a \in R\right.$ be an index of left ideal $\}$, then the following are equivalent:

1) If $0 \neq a \notin Y(R)$, then $l(r(a))=R a \oplus X_{a}$.

2) If $k \notin Y(R), a \in R$, then $l(a R \cap r(k))=\left(X_{k a}\right)_{l}+R k$ with $k a \epsilon R \backslash Y(R)$ and $\left(X_{k a}: a\right)_{l} \cap R k_{\subseteq} l(a)$ for all $a \in R$, where $\left(X_{k a}: a\right)_{l}=l(a R)$ if $k a=0$.

\section{Proof:}

The proof is similar to that of (Lemma 3.1, [5] )

An element $a \in R$ is called a right regular if $r(a)=0$ [8].

\section{Theorem 2.3}

Let $R$ is a right AWJCP-injective. Then:

1) Any right regular element of $R$ is left invertible.

2) $Y(R) \subseteq J(R)$.

3) If $P$ is a reduced principal right ideal of $R$, then $P=e R$ where $e^{2}=e \epsilon R$ and $(1-e) R$ is an ideal of $R$.

\section{Proof:}

1) Let $0 \neq a \in R$ such that $r(a)=0$. Then $a \notin Y(R)$ and so $l(r(a))=R a \oplus X_{a}$ where $X_{a}$ is a left ideal of $R$ ( $R$ is AWJCP-injective). Hence $R=l(0)=R a \oplus X_{a}$ since $r(a)=0$, thus there exists $r \in R, x \in X_{a}$ such that $1=r a+x, a=a r a+a x, a(1-r a)=a x \in R a \cap X_{a}=0$ so $(1-r a) \operatorname{er}(a)=0$. Therefore $R a=R$ and hence $a$ is a left invertible.

2) If $y \in Y(R)$ and $a \in R$, then, $r(1-a y)=0$ implies that $v(1-a y)=1$ for some $v \in R$ by (1). Hence $y \in J(R)$.

3) Let $P$ be a nonzero reduced principally a right ideal. Then $P=a R$ for some $a \in R$, since $a^{2} \notin Y(R), l\left(r\left(a^{2}\right)\right)=R a^{2} \oplus X_{a^{2}}$ for some a left ideal $X_{a^{2}}$ of $R$. Hence $r(a)=r\left(a^{2}\right)$ shows that

$R a \oplus X_{a}=l(r(a))=l\left(r\left(a^{2}\right)\right)=R a^{2} \oplus X_{a^{2}}, X_{a^{2} \subseteq{ }_{R}} R$. Then there exists $r \in R, x \in X_{a^{2}}$ such that $a=r a^{2}+x, a^{2}=a r a^{2}+a x$, $a x=(1-a r) a^{2} \in \mathrm{R} a^{2} \cap X_{a^{2}}=0, a^{2}=a r a^{2}$, $(1-a r) \epsilon l\left(a^{2}\right)=l(a)=0$, which implies that $a=\operatorname{ara}(P$ is reduced), where $P$ is generated by the idempotent $e=a r$. Also for any $b \in R$, $(\mathrm{eb}-\mathrm{ebe})^{2}=0$ implies $b=e b e$, where $e R(1-e)=0$. Therefore $R(1-e) \subseteq(1-e) R$.

\section{Lemma $2.4[8]$}

Let $R$ be a right APS-injective ring, then $J(R) \subseteq Y(R)$.

The following corollary follows immediately form Lemma 2.4 and Theorem 2.3. 


\section{Corollary 2.5}

If $R$ is a right AWJCP-injective and right APS-injective ring, then $J(R)=Y(R)$.

\section{Regularity of Right AWJCP-injective Rings}

A ring $R$ is called $\mathrm{PP}$, if for any $a \in \mathrm{R}, a \mathrm{R}$ is projective and $R$ is a right SPP, if for any $a \notin Y(R), a R$ is projective. Every PP ring is SPP. A ring $R$ is called quasi regular, if $a \in a R a$ for all $a \notin Y(R)$ [7].

A ring $\mathrm{R}$ is called a strongly regular, if for every $a \in R$ there exists $b \in R$ such that $a=a^{2} b .[10]$.

\section{Remark 1: [7]}

$R$ is regular if and only if $R$ is a right nonsingular and a right quasi regular.

\section{Proposition 3.1}

The following conditions are equivalent for a ring $R$ :

1) $R$ is a quasi regular ring.

2) $R$ is a right JCP-injective and a right SPP ring .

3) $R$ is a right AWJCP-injective and a right SPP ring.

\section{Proof:}

Obviously: $1 \rightarrow 2 \rightarrow 3$.

$3 \rightarrow 1$, Suppose that $R$ is a right AWJCP-injective and right SPP-ring. For any $0 \neq a \notin Y(R)$, there exists a left ideal $X_{a}$ of $R$ such that $\operatorname{lr}(a)=R a \oplus X_{a}$. Since $R$ is a right SPP, then $r(a)=e R$ with $e^{2}=e \in R$. Let $f=1-e$. Then $\operatorname{lr}(a)=R f$, and $f^{2}=f \in R$, and so $a=a f$ and $f=d a+x$ for some $d \in R$ and $x \in X_{a}$.

Thus $a f=a d a+a x, a-a d a=a x \in R a \cap X_{a}=0$, this shows that $a=a d a$, and so $R$ is aquasi regular.

\section{Corollary 3.2}

Let $R$ be a ring. Then $R$ is a regular ring if and only if $R$ is a right nonsingular, a right SPP, and an AWJCP-injective ring.

\section{Proof:}

It Follows from Proposition 3.1 and Remark 1.

\section{Lemma 3.3 [14]}

Suppose $M$ is a right R-module with $S=\operatorname{End}\left(M_{R}\right)$.

If $l_{M}\left(r_{R}(a)\right)=M a \oplus X_{a}$, where $X_{a}$ is a left S-submodule of $M_{R}$. Set $f: a R \rightarrow M$ a right R-homomorphism, then $f(a)=m a+x$ with $m \in M$, $x \in X_{a}$.

A right R-module $M$ is called almost nil-injective [13], if for any $k \in N(R)$, there exists an S-submodule $X_{k}$ of $M$ such that $l_{M} r_{R}(k)=M k \oplus X_{k}$ as left S-module ( $S=\operatorname{End}(M)$ ). If $R_{R}$ is almost nil-injective, then we call $R$ a right almost nil-injective ring. 


\section{Theorem 3.4}

Let $R$ be a right SPP ring. Then $R$ is a right AP-injective ring if and only if $R$ is a right AWJCP-injective and every simple singular right R-module is almost nil-injective.

\section{Proof:}

First, we show that $Y(R)=0$. Suppose that $Y(R) \neq 0$, then there exists $0 \neq b \in Y(R)$ such that $b^{2}=0$. We claim that $Y(R)+r(b)=R$. Otherwise, there exists a maximal right essential ideal $M$ of $R$ such that $Y(R)+r(b) \subseteq M$. Thus $R / M$ is almost nil-injective and $l_{R / M} r_{R}(b)=(R / M) b \oplus X_{b}$, for some a left ideal $X_{b}$ of $R / M$. Let $f: b R \rightarrow R / M$ be defined by $f(b r)=r+M$. Then $f$ is well defined R-homomorphism so there exists $r \in R, x \in X_{b}$ such that

$1+M=r b+M+x \quad($ Lemma $\quad 3.3), 1-r b+M=x \in(R / M) b \cap X_{b}=0$. Hence $1-r b \in M$. Since $r b \in Y(R) \subseteq M$, then $1 \epsilon M$, which is a contradiction. Therefore $Y(R)+r(b)=R$. Hence $1=c+d$ for some $c \epsilon Y(R)$ and $\operatorname{der}(b)$. Thus $b=b c, b(1-c)=0$. Since $c \in Y(R) \subseteq J(R)$ [Theorem $2.3(2)],(1-c)$ is invertible. Thus $b=0$, which is a contradiction. Hence $Y(R)=0$. By Corollary $3.2 R$ is a right AP-injective.

The converse is clear.

\section{Lemma 3.5 [5]}

If $R$ is a right AP-injective ring, then $J(R)=Y(R)$.

By Theorem 3.4 and Lemma 3.5 we get:

\section{Corollary 3.6}

Let $R$ be a right SPP ring. If $R$ is a right AWJCP-injective and every simple singular right $\mathrm{R}$-module is almost nil-injective, then $Y(R)=J(R)=0$.

\section{Theorem 3.7}

\section{Proof:}

Let $R$ be a right AWJCP-injective ring and right PP. Then $R$ is regular.

Let $0 \neq a \in R$. Then $a \notin Y(R)$ [Theorem 2.9,7]. Since $R$ is a right AWJCP-injective, then $l_{R} r_{R}(a)=R a \oplus X_{a}$ for some left ideal $X_{a}$ of $R$. Since $R$ is a right PP-ring $r(a)=r(e), e^{2}=e \epsilon R$.

Thus $R e=\operatorname{lr}(e)=\operatorname{lr}(a)=R a \oplus X_{a}$. Therefore $e=b a+x$ for some $x \in X_{a}$ and $b \in R$. So $a=a e=a b a+a x,(1-a b) a=a x \in R a \cap X_{a}=0, \quad$ and $a=a b a$. Hence $R$ is regular.

Following [1], a ring $R$ is called a left pseudo-morphic if for all $a \in R$ there exists $b \in R$ such that $R a=l(b)$. Every regular rings is pseudo-morghic.

From Theorem 3.7 have:

\section{Corollary 3.8}

Let $R$ be a right AWJCP-injective ring and a right PP. Then $R$ is a left pseudo-morphic.

A ring $R$ is called a left $N$ duo, if $R a$ is an ideal of $R$ for all $a \in N(R)$ [6]. 


\section{Lemma 3.9 [6]}

1) Let $R$ be a semiprime left $N$ duo ring. Then $R$ is reduced.

2) If $R$ is a reduced, then $Y(R)=Z(R)=0$.

\section{Proposition 3.10}

Let $R$ be a semiprime left $N$ duo ring, every simple singular right $\mathrm{R}$-module is AWJCP-injective. Then $R$ is a biregular ring.

\section{Proof:}

For any $a \in R, l(R a R)=r(R a R)=r(a)=l(a)$. If $R a R \oplus r(a) \neq R$, then there exists a maximal right ideal $M$ of $R$ such that $R a R \oplus r(a) \subseteq M$. If $M$ is not essential in $R$, then $M=r(e), e^{2}=e \epsilon R$. Therefore $e a=0$. Since $\mathrm{R}$ is a reduced $a e=0$. Hence $e \epsilon r(a) \subseteq r(e)$, which is a contradiction. So $M$ is essential in $R$. Since $R$ is a reduced (Lemma 3.9) $Y(R)=0$. Thus $R / M$ is AWJCP-injective, then $l_{R / M} r_{R}(a)=(R / M) a \oplus X_{a}, X_{a} \subseteq R / M$. defined.

Let $f: a R \rightarrow R / M$ be defined by $f(a r)=r+M$. Note That $f$ is well So $1+M=f(a)=c a+M+x, \quad c \in R, \quad x \in X_{a}, 1-c a+M=x \in R / M \cap X_{a}=0$, $1-c a \in M$. Since $c a \in R a R \subseteq M, 1 \in M$, which is a contradiction. Hence $R a R \oplus r(a)=R$ and so $R a R=e R, e^{2}=e \epsilon R$. Since $R$ is an abelian ring, $R$ is a biregular ring.

$R$ is called a right CAM-ring, if for any maximal essential right ideal $M$ of $R$ (if it exists) and for any right subideal $I$ of $M$ which is either a complement right subideal of $M$ or a right annihilator ideal in $R, I$ is an ideal of $M$ [10].

The right CAM-rings generalize semismple artinian. [10]

In [10], shows that semiprime right CAM-ring $R$ is either a semisiple artinian or a reduced.

A ring is called right MERT ring, if every maximal essential right ideal $M$ of $R$ is an ideal of $R$. [6]

The Following theorem is generalization of [Theorem 5.8, 7]

\section{Theorem 3.11}

The following are equivalent for a ring $R$ which is SPP

1) $R$ is either a semisimple artinain or a strongly regular ring.

2) $R$ is a semiprime, a right AWJCP-injective, a right CAM-ring.

3) $R$ is a semiprime, a right CAM-ring, a MERT ring every simple

\section{Proof:} singular right R-module is AWJCP-injective.

$1 \rightarrow \mathrm{i}, \mathrm{i}=2,3$ are obvious.

$2 \rightarrow 1$, if $R$ is not a semiprime artinian ring, then $R$ is reduced. By Corollary $3.2, R$ is a regular ring. Therefore $R$ is a strongly regular ring.

$3 \rightarrow 1$, if $R$ is not semisimple artinain ring, then $R$ is reduced. Let $0 \neq a \in R$. If $a R \oplus r(a) \neq R$. Then $a R \oplus r(a) \subseteq M$ for some maximal essential right ideal 
$M$ of $R$. Since $R$ is a reduced, then $Y(R)=0$. By a assumption, then simple singular right R-module $R / M$ is AWJCP-injective,

thus $l_{R / M} r_{R}(a)=(R / M) a \oplus X_{a}, X_{a} \subseteq R / M$. Let $f: a R \rightarrow R / M$ be defined by $f($ ar $)=r+M$.

Note that $f$ is well defined. Thus there exists $c \in R, x \in X_{a}$, such that $1+M=f(a)=c a+M+x$,

then $1-c a+M=x \in R / M \cap X_{a}=0,1-c a \in M$. But $c a \in M$ then $1 \epsilon M$, because $R$ is a MERT ring and $M$ is an ideal. It is a contradiction. Hence $a R \oplus r(a)=R$ and then $R$ is a strongly regular ring.

\section{REFERENCES}

[1] Camillo, V. and Nicholoson, W.K., (2015), "On rings where left principal ideal are left principal annihilators", Inter Electronic J. of Algebra. Vol. 17, pp. 199-214.

[2] Chen, J.L. and Ding, N.Q., (1999), "On general principally injective rings", Comm. Algebra, Vol. 27, pp. 2097-2116.

[3] Goodearl, K.R., (1979), "Von Neumann regular rings", Pitman (London).

[4] Nicholson, W.K. and Yousif, M.F., (1995), "Principally injective rings", J. Algebra, Vol. 174, pp. 77-93.

[5] Page, S.S. and Zhou, Y.Q., (1998), "Generalization of principally injective rings", J. Algebra, Vol. 206, pp. 706-721.

[6] Subedi, T., (2012), "On strongly regular rings and generalizations of semicommutative rings“, Inter. Math. Forum, Vol. 7, No. 16, pp. 777790.

[7] Wei, J.C., (2009), "JCP-injective rings", Int. Electron. J. of Algebra, Vol. 6, pp. 1-22.

[8] Yang Xiang, (2011), "Almost principally small injective rings", J. Kor. Math. Soc., Vol. 48, No. 6, pp. 1189-1201.

[9] Yue Chi Ming, R., (1976), "On annihilator ideals”, Math. J. Oka. Univ., Vol. 19, pp. 51-53.

[10] Yue Chi Ming, R., (1983), "On quasi frobeniusean and artinian rings", Pub. De Linstitut. Math. matique, 33(47), pp. 239-245.

[11] Yue Chi Ming, R., (1999), "A note on biregular rings", Kyngpook. Math. J., Vol. 39, pp. 165-173.

[12] Zhao, Yu-e and Du Xia, (2002), "Some studies on AP-injective rings", J. of Anhui Un.,Vo1. 26(1), pp. 7-11.

[13] Zhao, Yu-e and Du Xia, (2011), "On almost nil-injective rings", Inter Elec. J. of Algebra, Vo1. 9, pp. 103-113.

[14] Zhao, Yu-e, (2011), "On simple singular AP-injective modules", Inter. Math. froum. Vol. 6, No. 21, pp. 1037-1043. 